\title{
Normative blood pressure data in the early neonatal period
}

\author{
Alison L. Kent • Zsuzsoka Kecskes • Bruce Shadbolt • \\ Michael C. Falk
}

Received: 16 November 2006 /Revised: 28 February 2007 / Accepted: 5 March 2007 / Published online: 17 April 2007

(C) IPNA 2007

\begin{abstract}
There has been a temporal trend towards increased birth weight over the past three decades. This increase in birth weight may have resulted in an increase in neonatal blood pressure. Neonatal hypertension is becoming more common, especially in neonatal intensive care unit survivors. Current normative values are required to assist in diagnosis and appropriate management of neonatal hypotension and hypertension. The objective of this study was to determine normative blood pressure readings in healthy term neonates. Term neonates from the postnatal ward were enrolled from August 2003 to August 2005. Exclusion criteria included infants of mothers with preeclampsia, hypertension of any cause, gestational diabetes, type 1 diabetes mellitus and illicit substance use, infant congenital or chromosomal anomaly, admission to the neonatal intensive care unit or possible sepsis. Of the 406 infants enrolled, 218 were male. The median systolic, diastolic and mean blood pressures on day 1 of life were $65 \mathrm{mmHg}$, $45 \mathrm{mmHg}$, and $48 \mathrm{mmHg}$, respectively. On day 4, these values had increased to $70 \mathrm{mmHg}, 46 \mathrm{mmHg}$ and
\end{abstract}

\footnotetext{
A. L. Kent $(\bowtie) \cdot Z$. Kecskes

Department of Neonatology,

Centre for Newborn Care, The Canberra Hospital,

P.O. Box 11, Woden, Australian Capital Territory 2605, Australia

e-mail: alison.kent@act.gov.au

B. Shadbolt

Clinical Epidemiology Unit, The Canberra Hospital,

Canberra, Australian Capital Territory, Australia

M. C. Falk

Australian Capital Territory, Australia

A. L. Kent $\cdot$ Z. Kecskes $\cdot$ B. Shadbolt

Australian National University Medical School,

Canberra, Australian Capital Territory, Australia
}

$54 \mathrm{mmHg}$. There was a significant elevation in blood pressure from day 1 to day 2 of life. There was no significant difference in blood pressure readings with respect to birth weight or length. The only significant difference between the sexes was a lower mean and diastolic pressure on day 2 in boys. This study has provided current normative blood pressure readings of healthy term neonates that can be used to assess both hypotension and hypertension in the term neonate. No increase in blood pressure was noted from previous studies.

Keywords Blood pressure $\cdot$ Neonate $\cdot$ Hypertension

\section{Introduction}

Blood pressure (BP) measurements in the newborn infant are an important indicator of appropriate circulation. Determining whether a neonate is hypotensive or hypertensive requires normative BP values. There has been a temporal trend of increased birth weight over the past three decades [1]. However, no review has been made of potential changes in BP values during this time. Given that other studies have shown that BP in both preterm and term neonates is related to birth weight, a change in $\mathrm{BP}$ in neonates may have occurred over this time [2]. As BP increases within the first 5 days of life [3-8], an increase in birth weight may also result in an increase of BP values in the first days of life. Hypertension occurs in up to $2 \%$ of neonatal intensive care unit survivors [2]. Common causes include umbilical artery catheterisation, cardiac abnormalities, structural malformations of the kidney, renovascular or renal parenchymal disease and medications [9-15]. The long-term sequelae of hypertension in adults is well recognised; however, the long-term effects of neonatal 


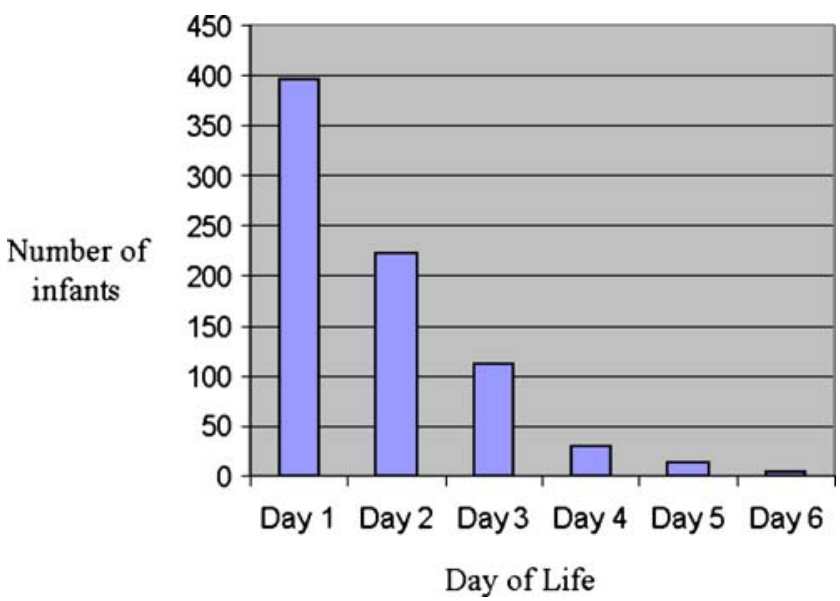

Fig. 1 Number of infants with blood pressure readings and postnatal day of life

hypertension are not clear. Detection and management of neonatal hypertension requires accepted normative values of BP. The majority of previous BP studies have (1) used infants admitted to the neonatal intensive care unit, (2) not excluded potential confounding maternal factors, (3) used ultrasound methods or (4) have included infants with a wide range of gestational age. The aim of this study was to determine normative BP values for hospital-based healthy term neonates on a postnatal ward using an automated BP technique, excluding potential confounding maternal or neonatal conditions.

\section{Methods}

All term infants ( $>36$ weeks gestation) delivered at The Canberra Hospital and admitted to the postnatal ward from August 2003 to August 2005 were eligible to be enrolled in the study. The local Human Research Ethics Committee approved the study. Written informed consent was obtained. Gestational age was determined by the obstetric staff from early ultrasound or last menstrual period. Infants of mothers with preeclampsia, hypertension of any cause, gestational diabetes, type 1 diabetes or a history of illicit substance use (i.e. methadone, heroin, marijuana), were excluded. Other exclusion criteria included congenital or chromosomal anomalies, infants with a birth weight less than the third percentile [16] and infants with possible sepsis or admission to the neonatal intensive care unit. Ethnicity was not collected, as indigenous and Asian populations constitute less than $10 \%$ of deliveries in this hospital.

BP measurements on day 1 were taken between 12 and $24 \mathrm{~h}$ of age to allow for resolution of fetal circulation. Three BP recordings were then taken each day between 0900 and 1200 until discharge home. Measurements were taken using an appropriate-sized BP cuff (the inflatable proportion of the cuff encircling $75 \%$ or greater of the limb circumference and the length of the cuff at least two thirds of the length of the upper limb segment) on an upper limb, with the infant in a resting state, awake or asleep. The Propaq Encore 202 EL monitor smartcuf noninvasive technology was used. Values were discarded if there was considerable movement of the infant. Averages of the three systolic, mean and diastolic readings were then used for analysis. Other information collected included maternal weight, BP at the 14-week gestation antenatal visit, maternal BP at delivery, Apgar scores, mode of delivery, gestation, gender, birth weight and length. A diagnosis of maternal hypertension of any cause, including pregnancy-induced hypertension, required at least three BP readings with systolic or diastolic measurements above the 95 th percentile. The single BP reading taken at the first antenatal visit and again on
Fig. 2 Systolic blood pressure percentiles (5th, 50th and 95th) on days 1-4 of life

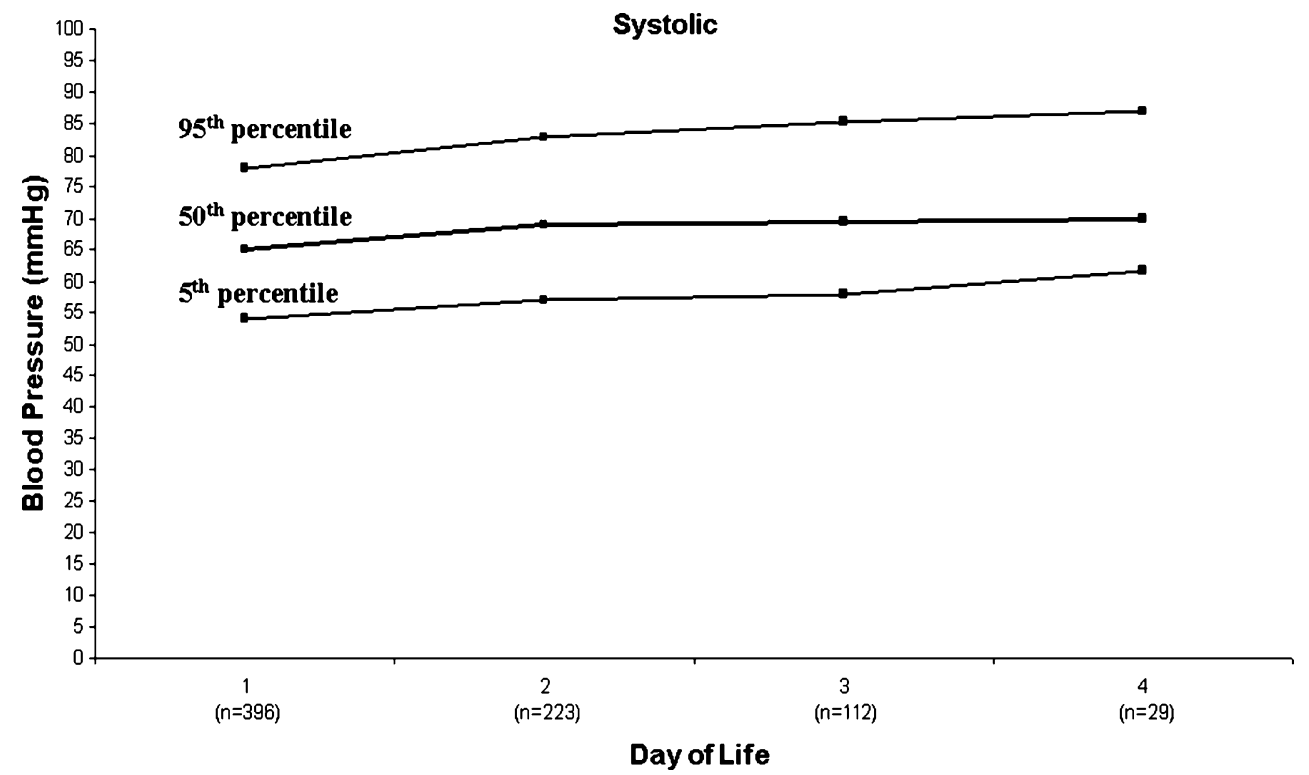


Fig. 3 Mean blood pressure percentiles (5th, 50th and 95th) on days 1-4 of life

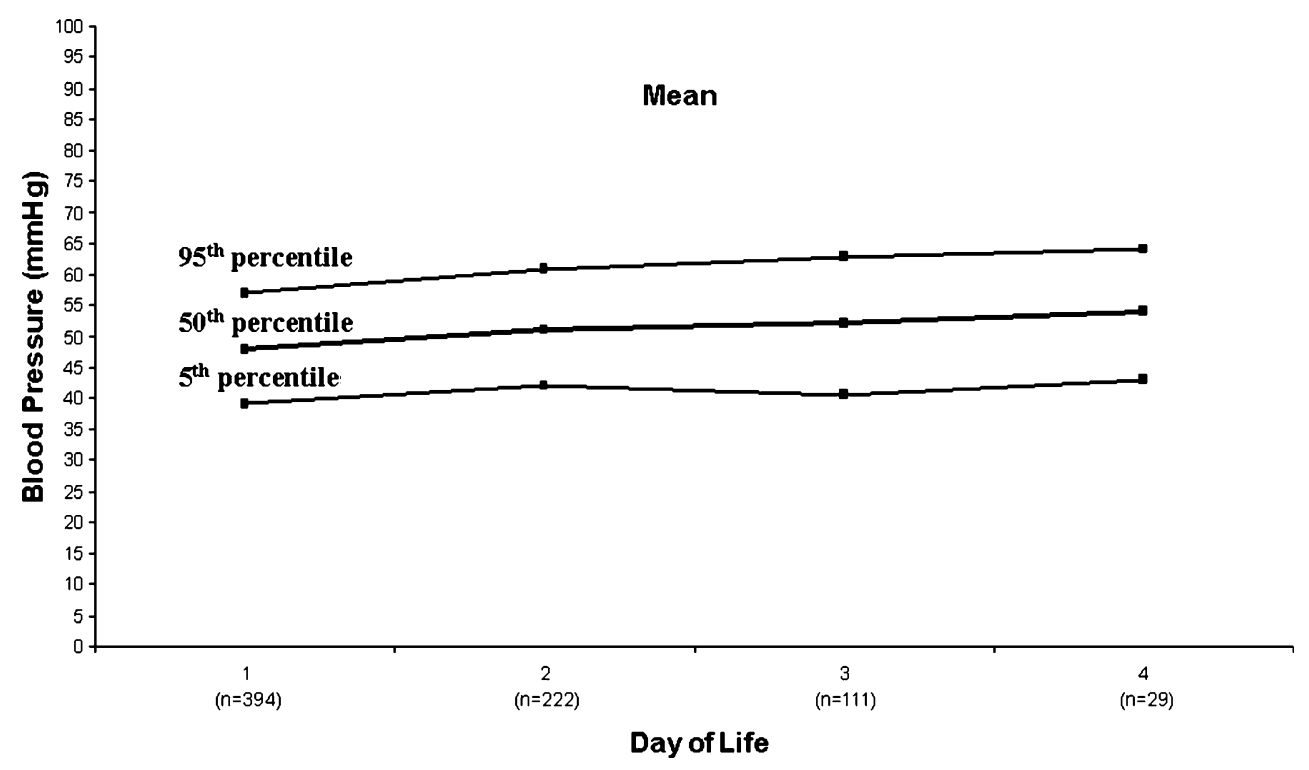

admission to the delivery suite prior to birth were noted for study purposes. If subsequent recordings resulted in a diagnosis of hypertension in the mother, the infant was excluded from the study. An Apgar score of $\geq 6$ at $5 \mathrm{~min}$ was considered normal, and admission to the neonatal intensive care unit for any reason, including low Apgar scores, was an exclusion criteria.

Data were stored and analysed using SPSS 12.0.1. Graphs were produced using SPSS 12.0.1 and Microsoft Excel 2000. Descriptive statistics were explored with medians, 5th and 95th percentiles and ranges reported for each of the average BP readings over time. Where babies' lengths and weights were reported, individualised BP averages over 2-4 days were used to handle the small sample sizes. There was no evidence that significant trends were lost by averaging over this period by weight and length. Wilcoxon signed rank tests were used to examine differences in BP readings over time. Kruskal-Wallis tests were used to examine differences between BP readings by weight and length stratified by day 1 and the individualised average over days 2-4. Spearman rank correlations were also carried out to explore trends between BP readings, weight and length for each day. Kruskal-Wallis tests were used to examine differences between BP and gender as well as gestation by week.

\section{Results}

A total of 406 infants were enrolled into the study. The median maternal weight was $65.0 \mathrm{~kg}$ (range 38-135). The median systolic BP at the first booking visit (14 weeks gestation) was $110 \mathrm{mmHg}$ (range 80-145) and diastolic BP was $60 \mathrm{mmHg}$ (range 42-90). At delivery, the median systolic BP was $120 \mathrm{mmHg}$ (range 90-160) and diastolic $\mathrm{BP}$ was $70 \mathrm{mmHg}$ (range 50-100). There were 218 males
Fig. 4 Diastolic blood pressure percentiles (5th, 50th and 95th) on days 1-4 of life

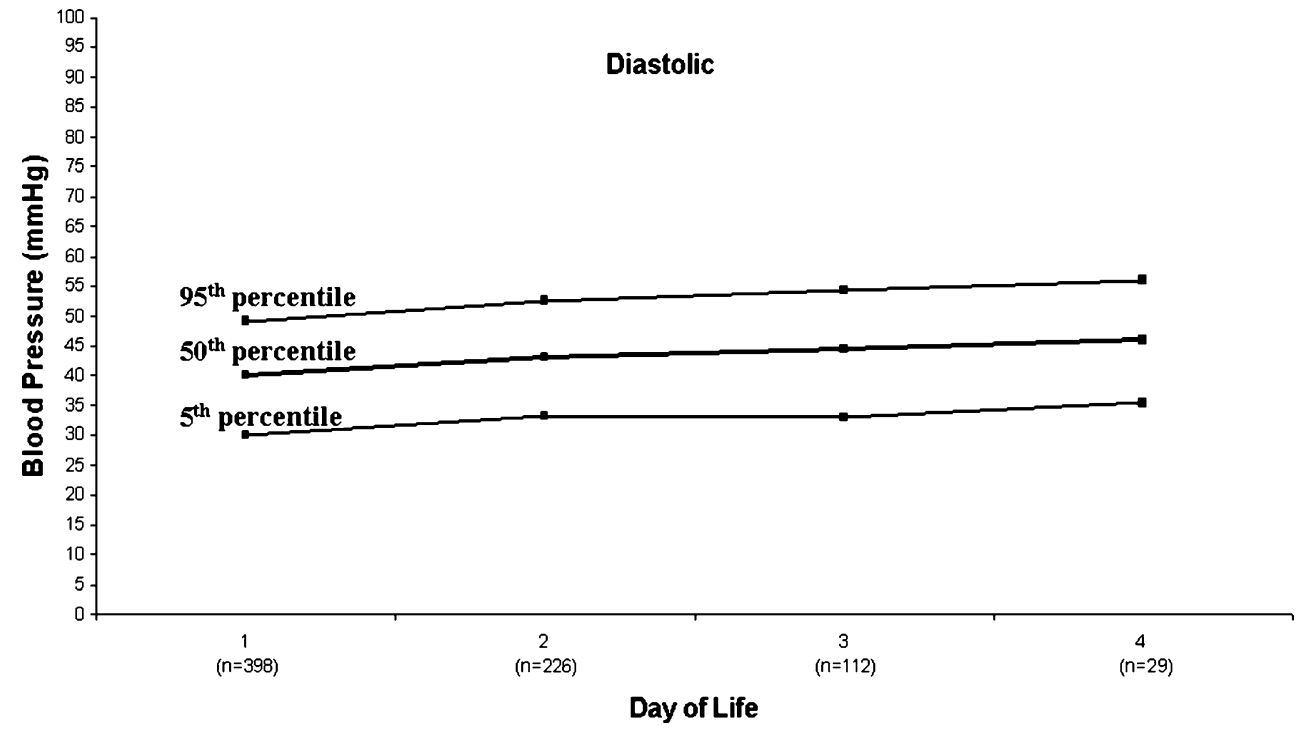


Fig. 5 Systolic, mean and diastolic blood pressures relative to birth weight on day 1 of life [the boxes show 5 th and 95 th confidence intervals (CI), the horizontal bar indicates the median and the longitudinal lines represent the range of values]. Blue $=$ diastolic, red $=$ mean, green $=$ systolic

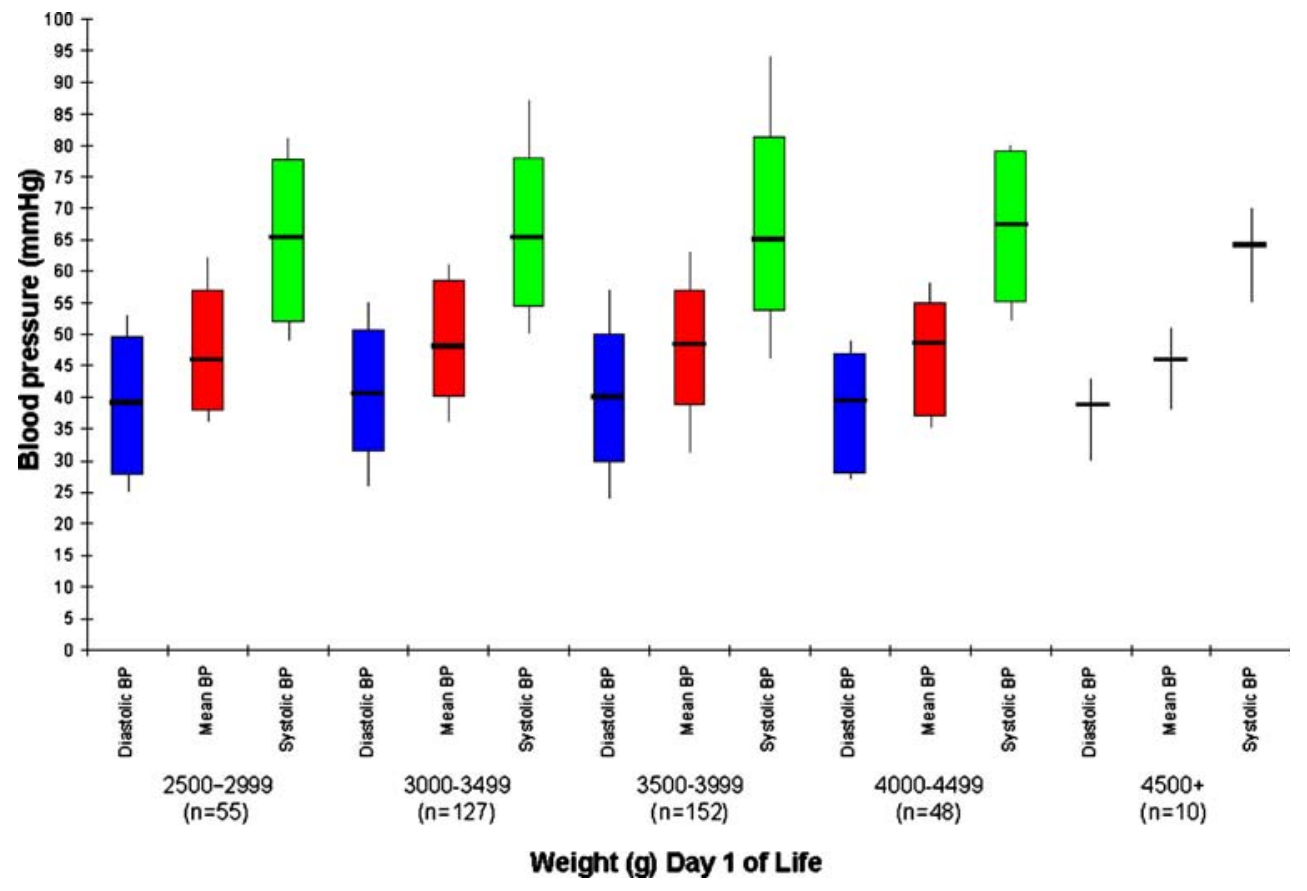

(53.7\%) and 188 females (46.3\%). The median gestation was 40 weeks (range 37-42). The median 1- and 5-min Apgar scores were 9 and 9, respectively (ranges 2-10; 6$10)$. Two hundred and sixty-five infants $(65 \%)$ were delivered vaginally, $48(12 \%)$ required instrumental assistance for vaginal delivery, $64(16 \%)$ were delivered by elective caesarean section and $29(7 \%)$ were delivered by emergency caesarean in labour. The median birth weight was $3,535.0 \mathrm{~g}$ (range 2,425-4,990), and the median length was $50.0 \mathrm{~cm}$ (range 41-57.5). The hospital's policy is that of early discharge with midwifery support at home. The numbers of infants with values available for each postnatal day are shown in Fig. 1. Due to the small number of values available on days 5 and 6 , these were not included in any analysis.

The median systolic, diastolic and mean BP (in $\mathrm{mmHg}$ ) on day 1 of life were 65,45 and 48 (ranges 46-94, 24-57, 31-63), respectively; on day 2, 68, 43 and 51 (ranges 4691, 27-58, 37-68), respectively; on day 3, 69.5, 44.5 and 52 (ranges 51-93, 26-61, 36-70), respectively; and on day $4,70,46$ and 54 (ranges 60-88, 34-57, 41-65), respectively. The 5th, 50th and 95th percentiles for systolic,

Fig. 6 Systolic, mean and diastolic blood pressures relative to birth weight on days 2-4 of life [the boxes show 5th and 95th confidence intervals (CI), the horizontal bar indicates the median and the longitudinal lines represent the range of values]. Blue $=$ diastolic, red $=$ mean, green $=$ systolic

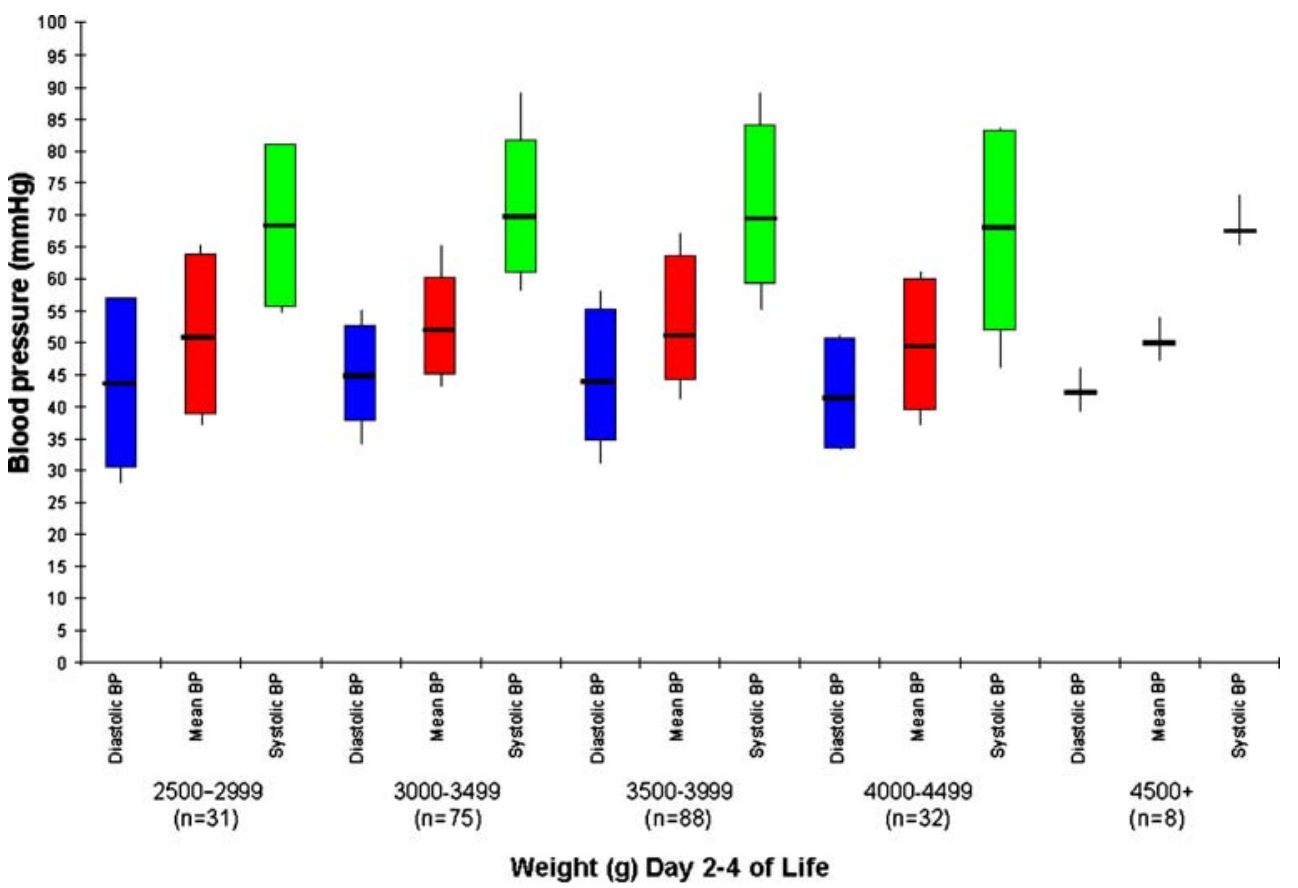


Fig. 7 Systolic, mean and diastolic blood pressures relative to birth length on day 1 of life [the boxes show 5th and 95th confidence intervals (CI), the horizontal bar indicates the median and the longitudinal lines represent the range of values]. Blue $=$ diastolic, red $=$ mean, green $=$ systolic

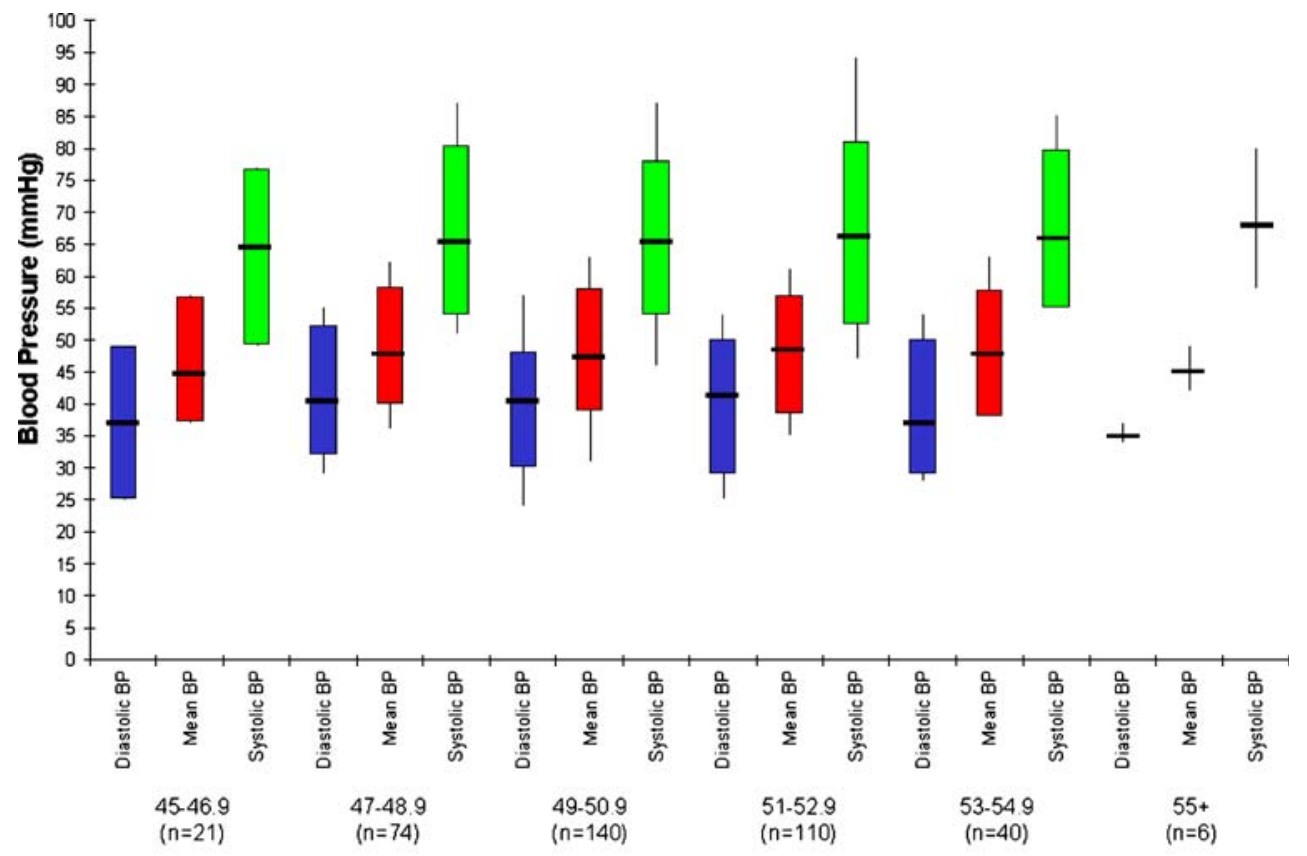

Length $(\mathrm{cm})$ Day 1 of Life diastolic and mean BPs on each day of life are shown in Figs. 2, 3 and 4. Systolic, diastolic and mean BP readings were all significantly higher on the second day of life from the first day of life ( $p$ values: $<0.001,<0.001,<0.001$, respectively). From day 2 to day 3 , there was no significant difference in systolic BP $(p=0.07)$, however, there was a significant rise in diastolic and mean $\mathrm{BP}(p<0.0001$ and $p=$ 0.005 , respectively). From day 3 to day 4 , there were no significant differences in systolic, mean or diastolic BP ( $p=$ $0.4, p=0.4, p=0.4$, respectively).
Systolic, diastolic and mean BP readings on day 1 of life and from day 2 to day 4 combined were analysed in respect to weight and length. There was no significant difference in $\mathrm{BP}$ readings with respect to birth weight (day $1 p=0.2$, days 2-4 $p=0.06$ ) or length (day $1 p=0.1$, days $2-4 p=0.05$ ) (Figs. 5, 6, 7 and 8).

Male infants had a significantly higher birth weight and length ( $p=0.007$ and $<0.001$, respectively). A lower mean and diastolic BP was seen in male infants on day $2(p=0.03$
Fig. 8 Systolic, mean and diastolic blood pressures relative to birth length on days 2-4 of life [the boxes show 5th and 95th confidence intervals (CI), the horizontal bar indicates the median and the longitudinal lines represent the range of values]. Blue $=$ diastolic, red $=$ mean, green $=$ systolic

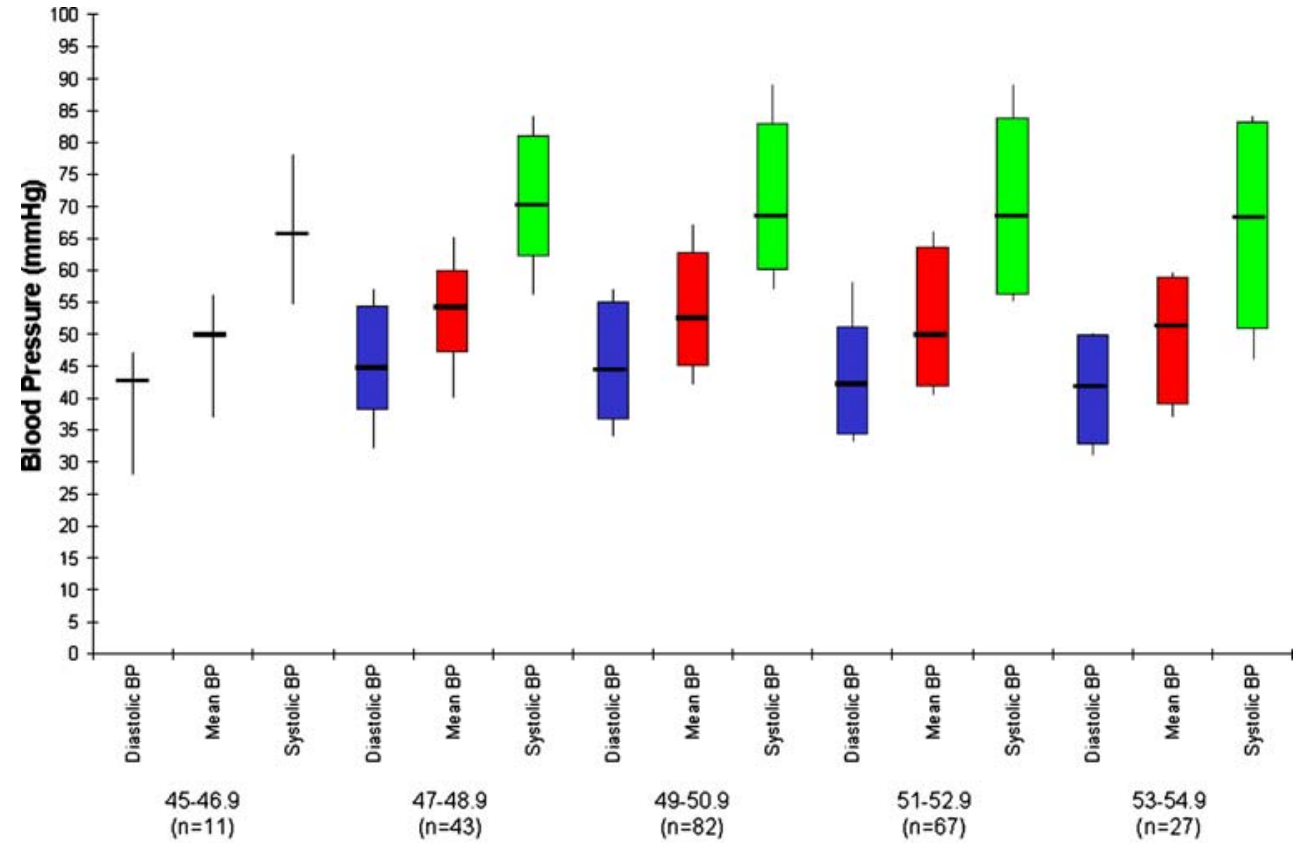

Length $(\mathrm{cm})$ Day 2.4 of Life 
and 0.01 , respectively). No significant differences in BP were noted for gestation week at delivery.

\section{Discussion}

This study provides current normative BP values for healthy term neonates. No previous study has used healthy infants on a postnatal ward without any confounding maternal or neonatal factors. This is also the first Australian study providing normative BP values in term neonates. A limitation of this study is the loss of infants after day 4 due to the early discharge policy. However, after day 2, there were no significant differences in BP readings over time. This study used an automated technique that removes the potential variations produced by the Doppler techniques described in previous studies.

De Swiet provided the first BP data for term neonates in 1976 [17]. This study looked at normal babies, however, only day 4 measurements were provided, and these were produced using a Doppler ultrasound and cuff, with only the systolic pressure provided. No infants with maternal confounders were excluded. The systolic pressures ranged from 50-100 mmHg. De Swiet provided further data in 1980 [18], but again, this study only included day 4 measurements and systolic readings. In their 1980 study, Uhari et al. included term infants who were not admitted to a nursery [6]. However, their study used a Doppler ultrasound method, and only provided systolic and diastolic readings. There were no exclusions for maternal confounders. The systolic range was between 60 and $80 \mathrm{mmHg}$ and the diastolic between 40 and $50 \mathrm{mmHg}$. Versmold et al., in 1981, provided some of the first umbilical arterial BP readings on neonates [19]. This study included only 45 infants, of whom 16 were less than 1,000 grams, and the readings were for the first $12 \mathrm{~h}$ of life. All of these infants required neonatal intensive care, and there were no exclusions for maternal confounders. The systolic range was between 50 and $80 \mathrm{mmHg}$, diastolic between 25 and $50 \mathrm{mmHg}$ and mean between 35 and $65 \mathrm{mmHg}$. The most quoted reference ranges for neonatal $\mathrm{BP}$ is from Zubrow et al. [7]. These were cuff measurements taken on days $1-5$ of life. Of the cases included, 280 were greater than 36 weeks gestation. All of these infants had been admitted to a neonatal intensive care unit with a variety of problems, and no maternal confounders were excluded. The systolic ranges were from 40 to $90 \mathrm{mmHg}$ and the diastolic from 25 to $60 \mathrm{mmHg}$. No mean BP were provided. Admission to a neonatal unit may be associated with stressful factors such as IV insertion, phototherapy and separation of the infant from his or her mother. Consequently, although these infants may be considered healthy, their BP may be elevated due to these concurrent stressors.
Our study, one of the largest to date, has not shown an increase in BP readings to go with the temporal trend in increased birth weight seen in the past 3 decades. However, it is difficult to compare this study with earlier work due to the noted differences in measurement methods and exclusion criteria. However, this study did not show any significant difference in BP associated with birth weight, which suggests that weight at this early stage may not be significant. Barker's hypothesis suggested that the in utero environment can predispose an infant to cardiovascular disease and hypertension later in life [20, 21]. To remove any potential confounders in BP variability, we excluded all infants of mothers with hypertension of any cause, gestational diabetes and insulin-dependent diabetes. This removed potential predisposition for elevated BP as well as the effects of any maternal medications.

In summary, the ranges of systolic and diastolic BP in our study are similar to those of previous authors, as were the differences of BPs noted from day 1 to day 2. However, our study did not show any major significant difference in BP depending on birth weight or length. This study provides current normative BP values, including mean readings that are frequently used in the neonatal intensive care environment, for healthy term neonates.

Acknowledgements We would like to acknowledge The Private Practice Fund of The Canberra Hospital for financial support for equipment and personnel. We would like to thank Sandy Meskell, Centre for Newborn Care Research Nurse, for her efforts in recruitment and follow-up.

\section{References}

1. Kramer MS, Morin I, Yang H, Platt RW, Usher R, McNamara H, Joseph KS, Wen SW (2002) Why are babies getting bigger? Temporal trends in fetal growth and its determinants. J Pediatr 141:538-542

2. Flynn JT (2000) Neonatal hypertension: diagnosis and management. Pediatr Nephrol 14:332-341

3. Bada HS, Korones SB, Perry EH, Arheart KL, Ray JD, Pourcyrous M, Magill HL, Runyan W 3rd, Somes GW, Clark FC, Tullis KV (1990) Mean arterial blood pressure changes in premature infants and those at risk of intraventricular haemorrhage. J Pediatr 117:607-614

4. Hulman S, Edwards R, Cen YQ, Polansky M, Falkner B (1991) Blood pressure in the first three days of life. J Perinatol 11:231-234

5. Spinazzola RM, Harper RG, De Soler M, Lesser M (1991) Blood pressure values in 500 to 760 gram birth weight infants in the first week of life. J Perinatol 11:147-151

6. Uhari M (1980) Changes in blood pressure during the first year of life. Acta Paediatr Scand 69:613-617

7. Zubrow AB, Hulman S, Kushner H, Flakner B (1995) Determinants of blood pressure in infants admitted to neonatal intensive care units: a prospective multicenter study. J Perinatol 15:470-479

8. Nuntnarumit P, Yang W, Bada-Ellzey HS (1999) Blood pressure measurements in the newborn. Clin Perinatol 26:981-996

9. Singh HP, Hurley RM, Myers TF (1992) Neonatal hypertension. Incidence and risk factors. Am J Hypertens 5:51-55 
10. Adelman RD (1978) Neonatal hypertension. Pediatr Clin North Am 25:99-110

11. Inglefinger JR (1982) Hypertension in the first year of life. In: Inglefinger JR (ed) Pediatric Hypertension, Philadelphia, Saunders pp 229-240

12. Arar MY, Hogg RJ, Aranty BS, Seikaly MG (1994) Etiology of sustained hypertension in children in the south western United States. Pediatr Nephrol 8:186-189

13. Friedman AL, Hustead VA (1987) Hypertension in babies following discharge from a neonatal intensive care unit. a 3-year follow-up. Pediatr Nephrol 1:30-34

14. Buchi KF, Siegler RL (1986) Hypertension in the first month of life. J Hypertens 4:525-528

15. Skalina ME, Kliegman RM, Fanaroff AA (1986) Epidemiology and management of severe symptomatic neonatal hypertension. Am J Perinatol 3:235-239
16. Roberts C, Lancaster P (1999) Australian national birthweight percentiles by gestational age. Med J Aust 170:114-118

17. De Swiet M, Fayers P, Shinebourne EA (1976) Blood pressure survey in a population of newborn infants. Br Med J 2:9-11

18. De Swiet M, Fayers P, Shinebourne EA (1980) Systolic blood pressure in a population of infants in the first year of life: The Brompton Study. Pediatrics 65:1028-1035

19. Versmold HT, Kitterman JA, Phibbs RH Gregory GA, Tooley WH (1981) Aortic blood pressure values during the first 12 hours of life in infants with birth weight 610 to 4,220 grams. Pediatrics $83: 240-243$

20. Barker DJ, Gluckman PD, Godfrey KM, Harding JE, Owens JA, Robinson JJ (1993) Fetal nutrition and cardiovascular disease in adult life. Lancet 341:938-941

21. Barker DJ (1995) Fetal origins of coronary heart disease. BMJ 311:171-174 